\section{Pankreaskarzinom}

\section{Versorgung verbesserungswürdig}

Das Vorgehen bei Pankreaskarzinomen in deutschen Zentren entspricht nicht immer den aktuellen Empfehlungen. Dies ergab eine Umfrage der Arbeitsgemeinschaft Internistische Onkologie (AIO) und der Chirurgischen Arbeitsgemeinschaft Onkologie (CAO).

Der Umfrage zufolge hielten nur $61 \%$ der teilnehmenden Zentren eine histologische Sicherung des Pankreaskarzinoms für notwendig, obwohl die S3Leitlinie eine bioptische Diagnosesicherung als obligat vorsieht, so Volker Heinemann, München [Adler G et al. Z Gastroenterol 2007;45:487-523]. Palliativ setzten $23,2 \%$ der Zentren eine Mono-Chemotherapie ein, obwohl laut S3-Leitlinie die Patienten mehr von einer Kombinationstherapie profitieren. Bei gutem Allgemeinzustand nutzen 59,1\% eine Kombinationstherapie. Diese Behandlung sollte bis zur Progression der
Erkrankung durchgeführt werden, betonte Heinemann. Nach eigenen Angaben tun dies aber nur $67 \%$ der Zentren.

Eine der zunehmend häufiger eingesetzten palliativen Kombinationstherapien ist die mit Gemcitabin und Erlotinib (Tarceva $\left.{ }^{\circledR}\right)$. Im Vergleich zu Gemcitabin allein erhöht die Kombination mit Erlotinib das Überleben signifikant [Moore MJ et al. J Clin Oncol 2007;25:1960-6]. Ein Zeichen für das Ansprechen auf die Therapie ist wie bei allen Wirkstoffen, die den epidermalen Wachstumsfaktor-Rezeptor hemmen, ein Hautausschlag (Rash). Obgleich dieser gut zu behandeln ist, ist es wichtig, die Patienten vor Behandlungsbeginn darüber aufzuklären. Einerseits, damit sie prophylaktisch die Haut pflegen, andererseits, damit rasch nach ihrem Auftreten die Hauterscheinungen spezifisch behandelt werden, erklärte Ralf Gutzmer, Hannover. In der Studie war das Gesamtüberleben von Patienten mit einer Hautreaktion vom Schweregrad 1 und mehr deutlich verlängert $(10,5$ Monate mit Rash vs. 5,3 Monate ohne Hautausschlag $(\mathrm{p}<0,001))$. Deshalb sollte bei Patienten, die nicht innerhalb der ersten vier bis acht Wochen einen solchen Ausschlag entwickeln, die Therapie mit Erlotinib überdacht werden.

Satellitensymposium im Rahmen des 29. Deutschen Krebskongresses am 25. Februar 2010 in Berlin; Veranstalter: Roche Pharma

\title{
Kolorektale Karzinome Patienten mit KRAS-Wildtyp profitieren von Panitumumab
}

Fast jeder zweite Patient mit kolorektalem Karzinom (mCRC) entwickelt Metastasen. Für diese Patienten ist Panitumumab, der erste voll humane Anti-EGFR-Antikörper, eine Option.

In zwei Phase-III-Studien wurde Panitumumab (Vectibix $\left.{ }^{\circledR}\right)$ in Kombination mit einer Chemotherapie beim mCRC untersucht. In der Studie „181“ wurde der

\section{Kurz notiert}

Bevacizumab zeigt positive Resultate bei Frauen mit fortgeschrittenem Eierstockkrebs In einer Phase-III-Studie verlängerte Bevacizumab (Avastin ${ }^{\circledR}$ ) plus Chemotherapie mit anschließender Bevacizumab-Erhaltungstherapie die Zeit bis zum Fortschreiten von nicht vorbehandeltem fortgeschrittenem Eierstockkrebs im Vergleich zur Chemotherapie allein. In der jetzt auf der ASCO-Jahrestagung vorgestellten Studie waren 1.873 Ovarialkarzinom-Patientinnen mit Beteiligung von Bauchfell und Eileitern behandelt worden [Burger RA et al. J Clin Oncol 2010;28:7s (suppl;abstr LBA1)].
Anti-EGFR-Antikörper zusammen mit FOLFIRI als Zweitlinien-Therapie eingesetzt. Diese Kombination verbesserte das mediane progressionsfreie Überleben bei Patienten mit nicht mutiertem KRAS-Gen (KRASWildtyp) signifikant um zwei Monate gegenüber FOLFIRI allein (5,9 vs. 3,9 Monate). Das mediane Gesamtüberleben verlängerte sich relativ ebenfalls um zwei Monate (14,5 vs. 12,5 Monate); doch dieser Unterschied fiel wegen einer hohen Crossover-Rate in den PanitumumabArm nicht signifikant aus. In der Studie „203“ wurde Panitu- mumab mit FOLFOX in der First Line kombiniert. Auch in dieser Studie profitierten Patietnen mit KRAS-Wildtyp: das mediane progressionsfreie Überleben verlängerte sich von 8,0 auf 9,6 Monate. Panitumumab ist in Europa zugelassen als Monotherapie zur Behandlung des metastasierten, EGFR-exprimierenden kolorektalen Karzinoms mit nichtmutiertem KRAS-Gen bei Patienten, bei denen fluoropyrimidin, oxaliplatin- und irinotecanhaltige Chemotherapieregime versagt haben.

Quelle: Satellitensymposium im Rahmen des 29. Deutschen Krebskongresses am 26. Februar 2010 in Berlin; Veranstalter: Amgen 